\title{
Bacillus Calmette-Guérin-stimulated Neutrophils Release Chemotaxins for Monocytes in Rabbit Pleural Spaces and In Vitro
}

\author{
Veena B. Antony, Steven A. Sahn, Asok C. Antony, and John E. Repine \\ Department of Medicine, Indiana University Medical Center, Indianapolis, 46223; Department of Medicine, University of South Carolina, \\ Charleston, South Carolina, 54238; and the Webb-Waring Lung Institute and Departments of Medicine and Pediatrics, University of \\ Colorado School of Medicine, Denver, Colorado 80262
}

\begin{abstract}
Neutrophils are often seen first at sites of granulomatous inflammation but their contribution to monocyte recruitment and granuloma formation is unknown. We tested the hypothesis that neutrophils release chemotaxins which attract monocytes. We found that rapid accumulations of fluid and influxes of neutrophils followed by monocytes occurred in bacillus Calmette-Guérin (BCG)-sensitized rabbits given BCG intrapleurally but did not occur in nitrogen mustard-treated (neutropenic) BCG-sensitized rabbits given BCG intrapleurally-unless the rabbits were also given intrapleural injections of neutrophils. We also found monocyte chemotaxins in pleural spaces of control and neutrophil-reconstituted neutropenic but not in neutropenic rabbits given BCG intrapleurally. Moreover, pleural fluid monocyte chemotaxins had molecular weights $(12,000-15,000$ and 1,000) that were similar to molecular weights of monocyte chemotaxins present in supernatants from mixtures of neutrophils and BCG in vitro. In addition, intrapleural injection of neutrophils and BCG or supernatants from in vitro mixtures of neutrophils and BCG (but not neutrophils or BCG alone) increased the numbers of monocytes and ${ }^{3} \mathrm{H}$ cell pellet activity in pleural fluids from untreated neutropenic rabbits or neutropenic rabbits previously injected intravenously with ${ }^{3}[\mathrm{H}]$ methyl thymidine-labeled monocytes. Furthermore, fewer BCG were recovered from pleural fluids of BCG-sensitized control compared to neutropenic rabbits given BCG, and at autopsy $10 \mathrm{~d}$ after instillation of BCG, control but not neutropenic rabbits had well-defined granulomas without adhesions on their pleural surfaces. Our results suggest that BCG stimulates neutrophils to release chemotaxins that recruit monocytes, and that these responses might contribute to granuloma formation in tuberculous pleurisy.
\end{abstract}

\section{Introduction}

Macrophages are characteristically seen in granulomas in tuberculosis and other chronic inflammatory diseases. These macrophages probably originate from circulating blood monocytes $(1,2)$, but the mechanisms responsible for their recruitment remain poorly defined (3). For example, although several inves-

Portions of this work have been presented at meetings of the Western Society for Clinical Investigation, American Thoracic Society, Aspen Lung Conference, and the American Federation for Clinical Research. Address reprint requests to Dr. V. B. Antony (111-P), VA Medical Center, 1481 West 10th St., Indianapolis, IN 46202.

Received for publication 12 March 1985

J. Clin. Invest.

(C) The American Society for Clinical Investigation, Inc.

$0021-9738 / 85 / 10 / 1514 / 08 \$ 1.00$

Volume 76, October 1985, 1514-1521 tigations have implicated $\mathrm{T}$ lymphocyte-derived chemotactic factors in attracting and localizing monocytes to foci of tuberculous infection (4), a dissociation exists between macrophage accumulation and lymphocyte-derived chemotactic activity in granulomatous inflammatory sites (5). Furthermore, in many other types of granulomatous inflammation, such as that produced by beryllium, development of delayed hypersensitivity does not appear necessary for production of granulomas. Because neutrophils are the first cells to appear in tuberculous exudates (6) and because neutrophils can release potent inflammatory mediators, we hypothesized that neutrophils might initiate inflammatory responses to tubercle bacilli by releasing chemotaxins which attract monocytes. Our results supported this premise. Briefly, we found that intrapleural instillation of bacillus Calmette-Guérin (BCG) caused an influx of monocytes into pleural fluids of control and neutrophil-reconstituted neutropenic but not into pleural fluids of neutropenic BCG-sensitized rabbits. In addition, after stimulation with BCG, neutrophils released chemotaxins for monocytes in vitro that had molecular weights that were similar to monocyte chemotaxins recovered from pleural spaces of control but not neutropenic BCG-sensitized rabbits given $B C G$ intrapleurally. Finally, intrapleural injection of neutrophils and BCG or supernatants from mixtures of neutrophils and BCG in vitro caused accumulations of monocytes or increased cell pellet ${ }^{3} \mathrm{H}$ activity in pleural fluids from untreated neutropenic rabbits or neutropenic rabbits previously injected intravenously with ${ }^{3}[\mathrm{H}]$ thymidine-labeled monocytes.

\section{Methods}

Purchase and care of rabbits. Healthy, New Zealand white rabbits ( 2.5 $\mathrm{kg}$ ) were purchased from a private rabbit supplier, fed standard rabbit laboratory diet and water ad lib, and kept in special quarters to avoid infection.

Recovery and purification of rabbit and human neutrophils. Blood for isolation of neutrophils was collected by arterial bleeding of rabbits or venipuncture of healthy, drug-free human subjects, placed in heparinized plastic syringes, and sedimented with dextran. Neutrophils were then purified by differential centrifugation on Ficoll-Hypaque $(7,8)$. Contaminating erythrocytes were removed by brief exposure to hypotonicity. Leukocyte pellets were then washed twice. This procedure yielded neutrophil-rich $(>95 \%)$ preparations that contained few lymphocytes $(4 \%)$ or monocytes $(1 \%)(7,8)$.

Recovery and purification of rabbit and human monocytes. Heparinized peripheral venous blood was centrifuged on Ficoll-Hypaque (9). The procedure yielded triply washed preparations that contained $>95 \%$ mononuclear cells.

Preparation of serum. Serum was obtained by venipuncture from rabbits or human donors, allowed to clot, rimmed, recovered by centrifugation, pooled, and frozen at $-70^{\circ}$ for no more than $1 \mathrm{mo}(10)$.

${ }^{3}[\mathrm{H}]$ methyl thymidine labeling of rabbit monocytes. Mononuclear cells were separated from the peripheral venous blood of BCG-sensitized rabbits. Monocytes were separated from lymphocytes by adherence to a 
glass petri dish for $1 \mathrm{~h}$. Monocytes (nonspecific esterase staining) were then incubated for $2.5 \mathrm{~h}$ at $37^{\circ} \mathrm{C}$ with ${ }^{3}[\mathrm{H}]$ methyl thymidine (New England Nuclear, Boston, MA) at a concentration of $200 \mu \mathrm{Ci} / 10^{6}$ mononuclear cells. Unincorporated methyl thymidine was removed by diluting the sample with $10 \mathrm{ml}$ of Hanks' balanced salt solution (HBSS) at $4^{\circ} \mathrm{C}$ and centrifuging at $1,000 \mathrm{~g}$ at $4^{\circ} \mathrm{C}$. This wash cycle was repeated four times. A final concentration of $1 \times 10$ cells $/ \mathrm{ml}$ of buffer was made.

Preparation of BCG. BCG (Glaxo, Inc., Research Triangle Park, NC) were obtained from the Trudeau Collection and plated fresh for each experiment on Middlebrook 7-H-11 medium (Difco Laboratories, Inc., Detroit, MI). For most studies, BCG were harvested and suspended in $0.9 \%$ saline at a concentration of $1 \times 10^{6} \mathrm{BCG}$ colony-forming units (CFU)' per $\mathrm{ml}$.

Sensitization of rabbits with $B C G$. New Zealand white rabbits are resistant to Mycobacterium tuberculosis but are susceptible and react to bovine forms of tubercle bacilli with responses characteristically seen in human infection with $M$. tuberculosis $(11,12)$. Rabbits were sensitized by injection of BCG $\left(1 \times 10^{6} \mathrm{CFU}\right)$ intradermally on the left leg. Immunized rabbits developed $0.4-1-\mathrm{mm}$ ulcerating lesions at injection sites within 1-12 d. Sensitization to BCG was confirmed 3 wk later by the finding of indurations of $10 \mathrm{~mm}$ or greater $48 \mathrm{~h}$ after administration of a challenge of $0.1 \mathrm{ml}$ of protein-purified derivative on the shaved skin of the right leg.

Production of BCG pleurisy in rabbits. Under aseptic conditions and local anesthesia with xylocaine, an 18-gauge catheter, capped with a rubber stopper to prevent air entry, was briefly placed percutaneously into the eighth intercostal space and BCG $\left(4 \times 10^{6} \mathrm{CFU}\right.$ suspended in $4 \mathrm{mi}$ of saline) were instilled into the pleural space $(13,14)$.

Analysis of pleural fluids. Thoracenteses were done percutaneously on the same rabbit for each determination (15). Aliquots of pleural fluid were spun at $2,500 \mathrm{~g}$, filtered through a nalgene filter $(0.2 \mu \mathrm{m})$, and then frozen at $-70^{\circ} \mathrm{C}$ for assay of chemotactic activity or analysis of ${ }^{3} \mathrm{H}$ activity. Differential cell counts on samples of pleural fluid were done by analyses of slides stained by modified Papanicolaou technique. Pleural fluid cells were characterized by using the following criteria (16). Neutrophils were identified by their typical multilobed nucleus and their coarse cytoplasmic pseudoeosinophilic granules. Monocytes were similar to peripheral blood monocytes. They measured $10-15 \mu \mathrm{m}$ in diameter, had nuclear cytoplasmic ratios of 1:2, were greyish-green, had cytoplasms without any inclusions, and were stainable with $\alpha$-napthyl acetate esterase. Macrophages were distinguishable from monocytes by being larger and having foamy vacuolated cytoplasms. Lymphocytes measured 8-10 $\mu \mathrm{m}$, had sharply demarcated margins, large nuclear-cytoplasmic ratios, and were not stainable with $\alpha$-napthyl acetate esterase.

Treatment of rabbits with nitrogen mustard. Neutropenia was induced in rabbits by pretreatment with a single dose of nitrogen mustard $(0.75$ $\mathrm{mg} / \mathrm{kg}$, Merck, Sharp \& Dohme Laboratories, West Point, PA) $60 \mathrm{~h}$ before intrapleural injection of BCG $\left(4 \times 10^{6} \mathrm{CFU}\right)(17)$. After treatment, neutrophil counts decreased to $<100 / \mathrm{mm}^{3}$ by $60 \mathrm{~h}$ and usually remained $<200 / \mathrm{mm}^{3}$ for the next $72 \mathrm{~h}$. In reconstitution experiments, normal neutrophils $\left(4 \times 10^{6}\right.$ in $4 \mathrm{ml}$ of saline) from control rabbits or supernatants $(4 \mathrm{ml})$ from mixtures of BCG and/or neutrophils in vitro were injected into pleural spaces of BCG-sensitized neutropenic rabbits.

Injection of rabbits with ${ }^{3}[\mathrm{H}]$ methyl thymidine-labeled rabbit monocytes and measurements of pleural fluid cell pellet ${ }^{3} \mathrm{H}$ activity. Immediately after intrapleural instillation of BCG and neutrophils, supernatants from mixtures of BCG, and neutrophils in vitro, BCG-sensitized rabbits were given intravenous injections of $2 \times 10^{7}$ rabbit monocytes which had been previously labeled with 30,000 counts of ${ }^{3}[\mathrm{H}]$ methyl thymidine. Pleural fluid was then obtained by serial thoracenteses and centrifuged at $1,000 \mathrm{~g}$ for $10 \mathrm{~min}$ at $4^{\circ} \mathrm{C}$. The supernatant was then aspirated, the cell pellet was resuspended in HBSS, and the sample was centrifuged at $1,000 \mathrm{~g}$. This cycle was repeated four times. Afterwards, the pleural fluid cell pellet was finally resuspended in $0.01 \mathrm{M} \mathrm{KPO}_{4}$, $\mathrm{pH} 7.5$, containing $1 \%$ Triton X-100. This sample was added to $10 \mathrm{ml}$ of 3 a 70 scintillation fluid (Research Products International Corp., Mount Prospect, IL) and

1. Abbreviation used in this paper: $\mathrm{CFU}$, colony-forming unit. counted in a Beckman Instruments LF 9800 scintillation counter (Palo Alto, CA) (60\% efficiency).

Incubation of $B C G$ and neutrophils in vitro. Washed purified human or rabbit neutrophils were suspended in $0.9 \%$ saline and incubated with optimal numbers of BCG (1:50 ratios) for optimal durations $(4 \mathrm{~h})$ at $37^{\circ} \mathrm{C}$. In some experiments, washed BCG that had been preopsonized by incubation in $50 \%$ serum for $0.5 \mathrm{~h}$ were used (18). To evaluate whether protein synthesis was necessary for chemotaxin production, cycloheximide $(20 \mu \mathrm{g} / \mathrm{ml})$ was added to mixtures of neurophils and BCG before or after the 4-h incubation (19). In some experiments, rabbit peripheral blood neutrophils were disrupted by repeated freeze thawing and fractions were extracted by ultracentrifugation. These fractions were evaluated for their chemotactic activity for monocytes.

Measurement of chemotactic activity. Chemotactic activity for monocytes was assessed using modified Boyden chambers equipped with Millipore filters ( $8 \mu \mathrm{m}$ pore size, Millipore Corp., Chicago, IL). Briefly, supernatants from pleural fluids, mixtures from neutrophils, and BCG in vitro or their controls were added to lower compartments of the chambers. Standard suspensions of $1.5 \times 10^{6}$ monocytes $/ \mathrm{ml}$ were then introduced into the upper compartments. The chambers were then incubated at $37^{\circ} \mathrm{C}$ in humidified air for $3 \mathrm{~h}$. After incubation, the filters were removed, fixed, and treated with $\alpha$-napthyl acetate esterase in order to identify monocytes (Sigma Chemical Co. Technical Bulletin No. 90, St. Louis, MO). After staining, filters were placed on glass cover slips, air dried, and mounted on glass slides. Chemotaxis was quantitated by counting the total number of monocytes in 10 high power oil immersion fields in 10 locations on each of three filters from separate experiments performed in triplicate. Chemotactic activity was expressed as the number of monocytes per oil immersion field reaching the lower surface of the filters. To determine whether migration of monocytes across the filters in response to supernatants was due to directed migration (chemotaxis) and/or increased random migration (chemokinesis), a checkerboard analysis was done by adding chemotactic fractions from supernatants to upper and lower compartments (20).

Characterization of chemotaxins for monocytes. Supernatants from pleural fluids, mixtures of BCG, and neutrophils in vitro or their controls were applied to Sephadex G-50 columns calibrated with ovalbumin (45,000 mol wt, Sigma Chemical Co.), cytochrome $c(12,500 \mathrm{~mol} \mathrm{wt}$ Sigma), and Bacitracin (1,450 mol wt, Pharmacia Fine Chemicals, Piscataway, $\mathrm{NJ}$ ) and eluted with HBSS. Eluted fractions were tested for their chemotactic activity for monocytes as a function of their molecular weights as described above. In some experiments, eluted fractions from supernatants from pleural fluids or in vitro mixtures of BCG and neutrophils were incubated for $24 \mathrm{~h}$ with trypsin (Sigma Chemical Co., 1 $\mathrm{mg} / \mathrm{ml}$ ), treated with Streptomyces griseus protease (Sigma Chemical Co., final concentration of $200 \mu \mathrm{g} / \mathrm{ml}$ ), or heated at $56^{\circ} \mathrm{C}$ for $30 \mathrm{~min}$ before assay for their chemotactic activity for monocytes. In some experiments, soybean trypsin inhibitor was added to inactivate trypsin prior to assay for chemotaxis.

Statistical analysis. Statistical analysis was done using analysis of variance and two-tailed Student's $t$ tests.

\section{Results}

Accumulation of fluid and inflammatory cells in pleural spaces of BCG-sensitized rabbits given intrapleural BCG. Intrapleural injection of BCG rapidly increased the volumes of fluid recovered from pleural spaces of BCG-sensitized rabbits. For example, $4.0 \pm 0.4,5.9 \pm 0.6,2.4 \pm 0.3,2.0 \pm 0.3$, or $1.8 \pm 0.4 \mathrm{ml}$ of fluid were recovered $4,24,48,72$, or $120 \mathrm{~h}$, respectively, from pleural spaces of BCG-sensitized rabbits injected intrapleurally with BCG. In contrast, throughout the 120 -h experiment, significantly $(P<0.05)$ smaller volumes of pleural fluid were obtained from BCG-sensitized rabbits that received intrapleural saline $(<1.0 \pm 0.4 \mathrm{ml})$, unsensitized rabbits that received intrapleural BCG $(<1.0 \pm 0.4 \mathrm{ml})$, or rabbits that received saline $(<0.4 \pm 0.2$ 


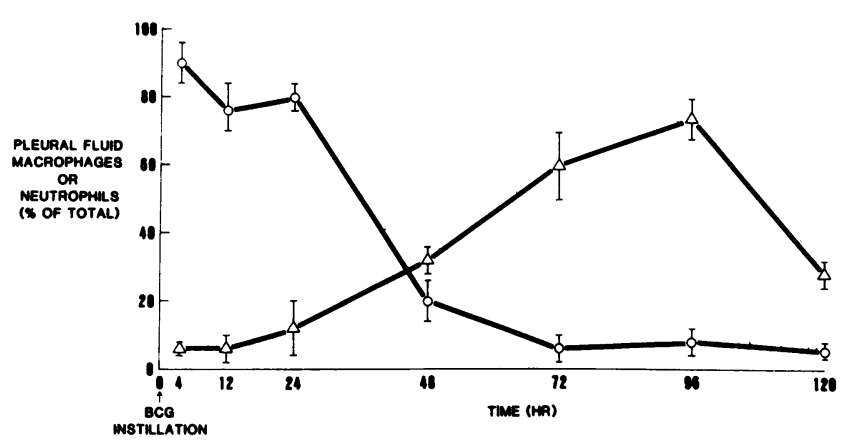

Figure 1. Accumulation of neutrophils and macrophages in pleural spaces of BCG-sensitized rabbits given BCG intrapleurally. After intrapleural instillation of BCG, neutrophils ( 0 ) accounted for $>75 \%$ of the total cells recovered from pleural spaces of BCG-sensitized rabbits for the first $24 \mathrm{~h}$; by $72 \mathrm{~h}$ after injection of BCG, macrophages $(\Delta)$ constituted $>60 \%$ of the cells. Each point is the mean \pm 1 SE of eight or more individual determinations.

$\mathrm{ml})$. In addition, more inflammatory cells were recovered from pleural spaces of BCG-sensitized rabbits given BCG intrapleurally. For example, $11 \pm 1.4,7.0 \pm 0.8,4.8 \pm 1.0,3.4 \pm 0.4$, or $3.2 \pm 0.5 \times 10^{3}$ cells/ $\mu$ l were recovered $4,24,48,72$, or 120 hours, respectively, from pleural spaces of $B C G$-sensitized rabbits injected intrapleurally with BCG. In contrast, throughout the 120 $\mathrm{h}$ period of the experiment, significantly fewer $(P<0.05)$ cells were obtained from pleural spaces of BCG-sensitized rabbits that received intrapleural saline $(<1.4 \pm 0.2)$, unsensitized rabbits that received intrapleural $\mathrm{BCG}(<3.1 \pm 0.4$ and $<1.2 \pm 0.3$ from 24 to $120 \mathrm{~h}$ ), or unsensitized rabbits that received saline $(<1.2 \pm 0.8)$. For the first $24 \mathrm{~h}$ after injection of BCG, neutrophils were the predominant cell type recovered from pleural spaces, accounting for $>75 \%$ of the total cells (Fig. 1). After $72 \mathrm{~h}$, macrophages constituted $60 \%$ of the total cells recovered from pleural spaces of BCG-sensitized rabbits given intrapleural BCG. By $120 \mathrm{~h},>70 \%$ of the cells recovered from pleural spaces were small lymphocytes. Lymphocytes constituted $90 \%$ of the total cells recovered from pleural fluids of all other groups.

Accumulation of fluid and inflammatory cells in pleural spaces of neutropenic BCG-sensitized rabbits given intrapleural $B C G$. Accumulation of pleural fluid and inflammatory cells was decreased $(P<0.05)$ in nitrogen mustard-pretreated (neutropenic) BCG-sensitized rabbits given BCG intrapleurally. For example, after intrapleural injection of $B C G, 1.5 \pm 0.2,1.7 \pm 0.3$, $1.2 \pm 0.2,1.0 \pm 0.1$, or $0.7 \pm 0.2 \mathrm{ml}$ of fluid was recovered from pleural spaces $4,24,48,72$, or $120 \mathrm{~h}$, respectively, after intrapleural injection of BCG into BCG-sensitized neutropenic rabbits. In addition, fewer $(P<0.05)$ inflammatory cells were obtained from pleural spaces of neutropenic rabbits given BCG. For example, $1.5 \pm 0.2,0.8 \pm 0.4,1.0 \pm 0.2,0.8 \pm 0.2$, or $0.6 \pm 0.2$ $\times 10^{3}$ cells/ml were obtained $4,24,48,72$, or $120 \mathrm{~h}$, respectively, from pleural spaces of neutropenic rabbits injected intrapleurally with BCG. At $72 \mathrm{~h}$ after intrapleural injection of BCG, neutropenic rabbits had fewer $(P<0.05)$ macrophages in their pleural spaces than control or neutropenic, neutrophil-reconstituted rabbits (Fig. 2). Nitrogen mustard-treated, unsensitized rabbits where pleural spaces were reconstituted with neutrophils showed $7 \pm 2 \%, 18 \pm 2 \%$, or $22 \pm 4 \%$ macrophages at 4,24 , or $48 \mathrm{~h}$, respectively. Lymphocytes were the most common cell ( $>95 \%$ of the total cells) recovered from pleural fluids of neutropenic, BCG- sensitized rabbits given BCG intrapleurally. The absence of macrophages in pleural spaces of neutropenic rabbits given BCG did not appear to be due to decreases in available monocytes. First, numbers of circulating monocytes were comparable $(P>0.05)$ in neutropenic and control rabbits. Second, when neutrophils were purified from the peripheral blood of BCGsensitized rabbits and instilled immediately after BCG into pleural spaces of neutropenic rabbits, increased numbers of macrophages were recovered from pleural fluids (Fig. 2). In addition, monocytes isolated from the peripheral blood of neutropenic rabbits or monocytes treated with nitrogen mustard in vitro had normal chemotactic responses toward zymosan-activated serum in vitro (data not shown).

Chemotactic activity for monocytes in pleural fluids from rabbits given intrapleural $B C G$. The aforementioned suggestion that neutrophils might release chemotaxins that recruit monocytes to pleural spaces was supported when increased $(P<0.05)$ chemotactic activity for monocytes was found in supernatants from pleural fluids from control and neutrophil-reconstituted but not neutropenic rabbits given BCG (Fig. 3). Moreover, monocyte chemotactic activity in supernatants from pleural fluids from BCG-sensitized rabbits given BCG peaked at $24 \mathrm{~h}$ and correlated with numbers of neutrophils recovered from pleural spaces (Fig. 4). Eluted fractions from supernatants from pleural fluids (obtained at maximal chemotactic activities $24 \mathrm{~h}$ after intrapleural injection of BCG) were chemotactic and chemokinetic by checkerboard analysis (Table I).

Chemotactic activity for monocytes in supernatants from mixtures of neutrophils and BCG in vitro. Supernatants from mixtures of BCG and neutrophils in vitro had increased $(P<0.05)$ chemotactic activity for monocytes compared to supernatants from neutrophils or BCG alone (Fig. 5). Eluted fractions from supernatants from in vitro mixtures of neutrophils and BCG (obtained at maximal chemotactic activities after incubation for $4 \mathrm{~h}$ ) were chemotactic and chemokinetic by standard checkerboard analysis (Table II). Incubation of neutrophils and BCG with cycloheximide or pretreatment of neutrophils with

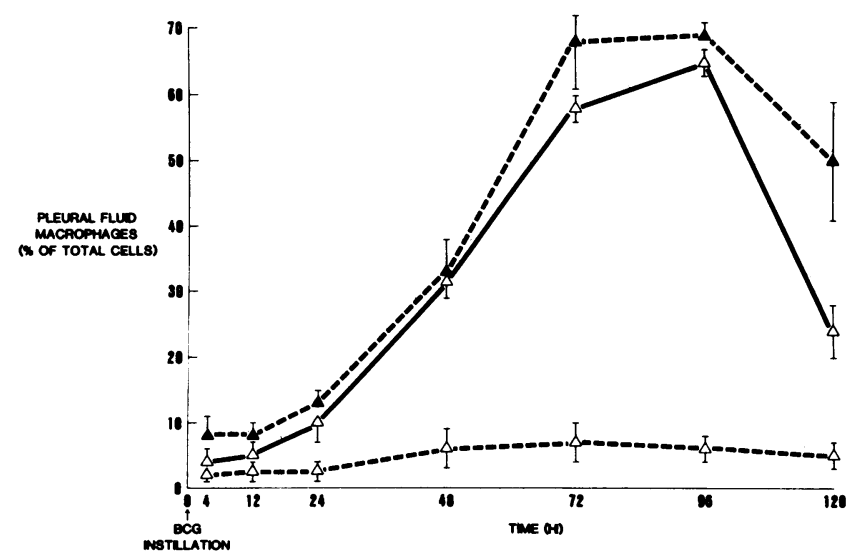

Figure 2. Accumulation of macrophages in pleural spaces of neutropenic or neutrophil-reconstituted neutropenic BCG-sensitized rabbits given BCG intrapleurally. After intrapleural instillation of BCG, significantly greater $(P<0.05)$ numbers of macrophages were recovered from BCG-sensitized control $(\Delta-\Delta)$ and neutropenic rabbits whose pleural spaces were reconstituted with neutrophils $(\Delta)$ than from pleural spaces of nonreconstituted BCG-sensitized neutropenic rabbits $(\Delta---\Delta)$. Each point is the mean \pm 1 SE of eight or more individual determinations. 


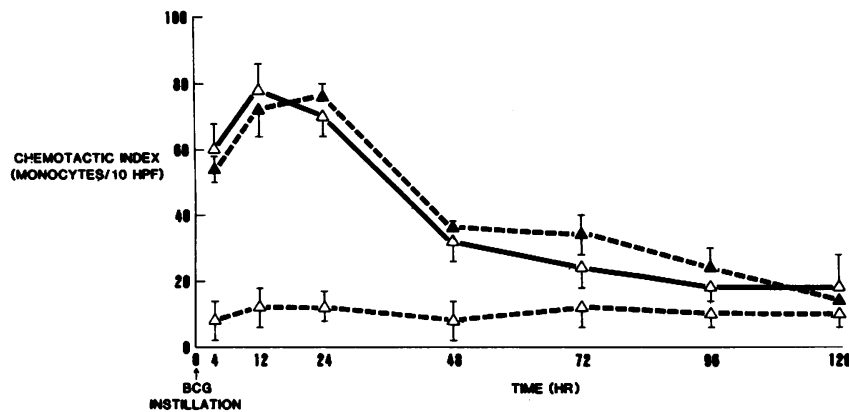

Figure 3. Monocyte chemotactic activity of supernatants from pleural fluids from BCG-sensitized rabbits given BCG intrapleurally. After intrapleural instillation of BCG, pleural fluid chemotactic activity for monocytes was greater $(P<0.05)$ in supernatants from BCG-sensitized control rabbits $(\Delta-\Delta)$ and BCG-sensitized neutropenic rabbits with pleural spaces reconstituted with neutrophils $(\Delta---\Delta)$ than in supernatants from nonreconstituted BCG-sensitized neutropenic rabbits $(\Delta---\Delta)$. Each point is the mean \pm 1 SE of eight or more individual determinations. HPF, high power field.

cycloheximide decreased $(P<0.05)$ production of chemotaxins for monocytes (Fig. 6). However, addition of cycloheximide after incubation of BCG and neutrophils in vitro did not decrease the chemotactic activity for monocytes of these supernatants. The latter suggests that cycloheximide does not directly interfere with already formed chemotaxins for monocytes (Fig. 6).

Chemotaxins for monocytes in supernatants from pleural fluids (in vivo) or mixtures of neutrophils and BCG in vitro had similar molecular weights (Fig. 7). In both cases, two areas of chemotactic activity for monocytes were prominent. One was a relatively small molecule of $<1,000 \mathrm{~mol} \mathrm{wt}$; the other was between 12,000 and 15,000 mol wt. Addition of cycloheximideinhibited production of both fractions and chemotactic activity. When diluted by $50 \%$, eluted in vivo and in vitro fractions had significantly $(P<0.05)$ more chemotactic activity for monocytes (respectively, $63 \pm 1 \%$ and $62 \pm 5 \%$ of chemotactic responses produced by zymosan-activated serum) than for neutrophils ( $14 \pm 6 \%$ and $9 \pm 3 \%$, respectively). When diluted to $5 \%$, fractions from

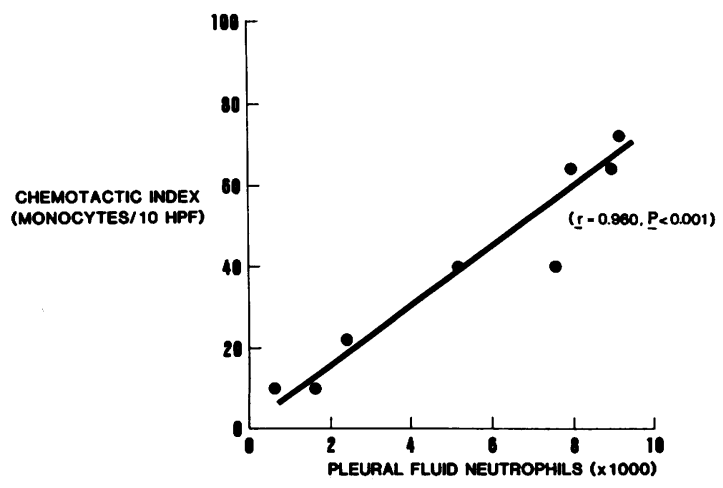

Figure 4. Relationship between monocyte chemotactic activity and numbers of neutrophils in pleural fluids from BCG-sensitized rabbits given BCG intrapleurally. Pleural fluid chemotactic activity for monocytes correlated $(P<0.001, r=0.960)$ with the absolute numbers of neutrophils recovered from pleural spaces of BCG-sensitized rabbits given intrapleural BCG. Each point is the mean \pm 1 SE of eight or more individual determinations. HPF, high power field.
Table I. Migration of Rabbit Blood Monocytes In Vitro toward Chemotactic Fractions from Supernatants of Pleural Fluids from BCG-sensitized Rabbits Given Intrapleural BCG*

\begin{tabular}{llllll}
\hline $\begin{array}{l}\text { Concentration of } \\
\text { supernatant } \\
\text { below filter }\end{array}$ & \multicolumn{5}{l}{ Concentration of supernatant above filter (\%) } \\
\cline { 2 - 6 }$\%$ & 0 & 25 & 50 & 75 & 100 \\
\hline$\%$ & \multicolumn{2}{l}{ migrated cells/10 HPF } & & & \\
0 & $12 \pm 6 \neq$ & $19 \pm 4$ & $18 \pm 6$ & $16 \pm 8$ & $17 \pm 6$ \\
25 & $42 \pm 6$ & $26 \pm 5$ & $27 \pm 2$ & $24 \pm 7$ & $21 \pm 5$ \\
50 & $61 \pm 3$ & $44 \pm 2$ & $28 \pm 4$ & $25 \pm 4$ & $20 \pm 3$ \\
75 & $74 \pm 8$ & $66 \pm 5$ & $53 \pm 3$ & $29 \pm 2$ & $23 \pm 5$ \\
100 & $88 \pm 7$ & $75 \pm 6$ & $58 \pm 7$ & $49 \pm 6$ & $38 \pm 4$ \\
\hline
\end{tabular}

HPF, high power field.

* The diagonal column represents migration of monocytes in the absence of a chemoattractant gradient (chemokinesis). The values below the diagonal represent migration in the presence of a positive gradient (chemotaxis).

$\ddagger$ Values given \pm 1 SEM.

pleural fluids or in vitro mixtures still had less chemotactic activity for neutrophils $(5 \pm 3 \%$ or $2 \pm 1 \%$, respectively) whereas chemotactic activity for monocytes remained at $45 \pm 7 \%$ or $49 \pm 8 \%$, respectively. Supernatants from sonicated neutrophils from control or BCG-sensitized rabbits did not contain significant chemotactic activity $(14 \pm 3 \%)$ for monocytes compared to supernatants from mixtures of neutrophils and BCG in vitro $(65 \pm 12 \%)$. More macrophages were recovered from pleural fluids from neutropenic rabbits injected intrapleurally with supernates from mixtures of BCG and neutrophils in vitro than from rabbits injected with supernatants from in vitro mixtures of heat-killed neutrophils and BCG, neutrophils or BCG (Fig. 8). Eluted frac-

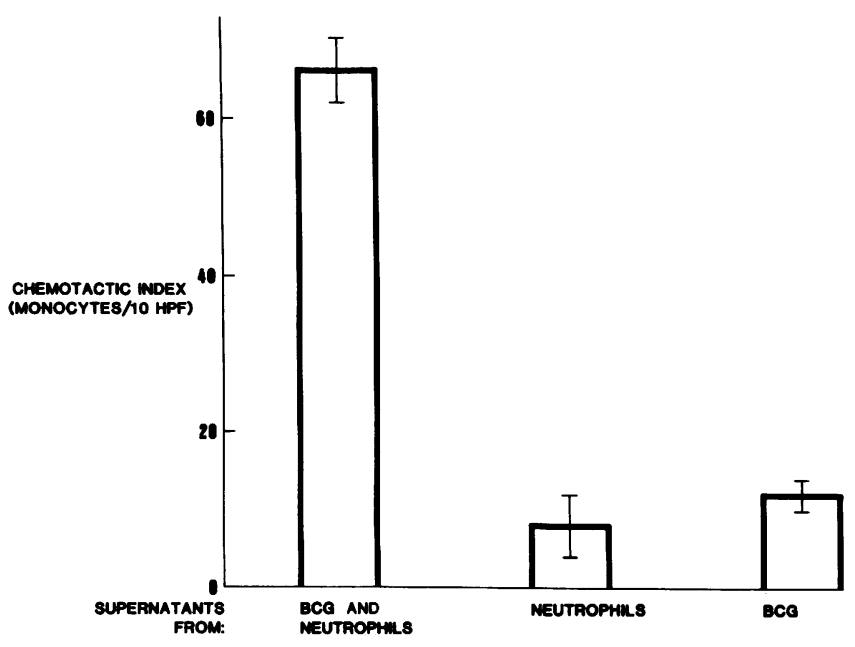

Figure 5. Monocyte chemotactic activity in supernatants from mixtures of neutrophils and BCG in vitro. Supernatants diluted $50 \%$ in HBSS from in vitro mixtures of BCG and neutrophils in vitro had significantly more $(P<0.01)$ chemotactic activity for monocytes than supernatants from mixtures of BCG or neutrophils alone. Each point is the mean $\pm \mathrm{SE}$ of six to eight individual determinations. HPF, high power field. 
Table II. Migration of Rabbit Blood Monocytes In Vitro toward Chemotactic Fractions Eluted from Supernatants of Mixtures of BCG and Neutrophils In Vitro*

\begin{tabular}{|c|c|c|c|c|c|}
\hline \multirow{2}{*}{$\begin{array}{l}\text { Concentration of } \\
\text { supernatant } \\
\text { below filter }\end{array}$} & \multicolumn{5}{|c|}{ Concentration of supernatant above filter (\%) } \\
\hline & 0 & 25 & 50 & 75 & 100 \\
\hline \% & migrate & $/ 10 \mathrm{HPF}$ & & & \\
\hline 0 & & & $16 \pm 3$ & $16 \pm 7$ & $13 \pm 6$ \\
\hline 25 & $37 \pm 7$ & & & $20 \pm 3$ & $19 \pm 5$ \\
\hline 50 & $59 \pm 6$ & & & & $24 \pm 3$ \\
\hline 75 & $73 \pm 4$ & $64 \pm 5$ & & & \\
\hline 100 & $80 \pm 7$ & $77 \pm 8$ & $62 \pm 7$ & & \\
\hline
\end{tabular}

HPF, high power field.

* The diagonal column represents migration of monocytes in the absence of a chemoattractant gradient (chemokinesis). The values below the diagonal represent migration in the presence of a positive gradient (chemotaxis).

$\ddagger$ Values given \pm 1 SEM.

tions from supernatants from pleural fluids or in vitro mixtures of BCG and neutrophils that were subsequently incubated for $24 \mathrm{~h}$ with trypsin, treated with proteases from Streptomyces griseus, or heated at $56^{\circ} \mathrm{C}$ for $30 \mathrm{~min}$ did not have chemotactic activity for monocytes.

Accumulation of ${ }^{3} \mathrm{H}$ in cell pellets from pleural fluids from $B C G$-sensitized neutropenic and control rabbits previously injected with ${ }^{3} \mathrm{H}$-labeled blood monocytes. After intravenous injection of ${ }^{3} \mathrm{H}$-labeled monocytes, higher $(P<0.05){ }^{3} \mathrm{H}$ counts were found in cell pellets recovered from pleural fluids of BCGsensitized control rabbits given intrapleural BCG than from neutropenic rabbits given intrapleural BCG or control rabbits given intrapleural saline (Fig. 9). In parallel, neutropenic rabbits with pleural spaces reconstituted with neutrophils and BCG or with supernatates from mixtures of neutrophils and BCG in vitro had pleural fluid ${ }^{3} \mathrm{H}$ counts that equaled those seen in control rabbits given intrapleural BCG (Fig. 9).
BCG clearance, granuloma, and adhesion formation in pleural spaces of rabbits given $B C G$ intrapleurally. More $(P<0.05)$ BCG were recovered from pleural fluids of neutropenic than from control BCG-sensitized rabbits injected intrapleurally with BCG. For example, samples of pleural fluids from BCG-sensitized neutropenic rabbits, grew $40 \pm 8,50 \pm 6,56 \pm 4$, $59 \pm 7$, or $64 \pm 10$ BCG colonies/ml of pleural fluid $4,24,48,72$, or $120 \mathrm{~h}$, respectively, after injection of BCG intrapleurally. In contrast, samples of pleural fluid from BCG-sensitized conrol rabbits grew $44 \pm 5,37 \pm 1,34 \pm 3,31 \pm 2$, or $30 \pm 3$ BCG colonies/ $\mathrm{ml}$ of pleural fluid at $4,24,48,72$ or $120 \mathrm{~h}$, respectively, after injection of BCG intrapleurally. In addition, at autopsy $10 \mathrm{~d}$ after intrapleural injection of BCG, control rabbits had welldefined granulomas but no adhesions on their visceral and parietal pleura. In contrast, neutropenic rabbits given BCG did not have well-formed granulomas but had pleural surfaces that were covered by a fibrinous exudate and many adhesions. Microscopic examination confirmed these findings showing granulomas on pleural surfaces of control but not neutropenic BCGsensitized rabbits given BCG. Unsensitized rabbits given BCG, BCG-sensitized rabbits given saline, or unsensitized rabbits given saline did not have granulomas on their pleural surfaces or pleural adhesions.

\section{Discussion}

This study evaluated the contribution of neutrophils to monocyte recruitment and granulomatous inflammation seen in tuberculous pleurisy. Specifically, we tested the premise that exposure to BCG stimulates neutrophils to release chemotaxins that recruit monocytes and that these responses participate in granuloma formation. Considerable evidence was obtained to support this hypothesis. Large influxes of neutrophils preceded influxes of monocytes into pleural spaces of BCG-sensitized rabbits given BCG intrapleurally but did not occur in nitrogen mustard-pretreated (neutropenic) rabbits injected intrapleurally with BCG. The observed decreased influx of monocyte in neutropenic rabbits was not a direct effect of nitrogen mustard on monocytes in that after direct reconstitution of pleural spaces with neutrophils, monocyte accumulation occurred normally in neutropenic rabbits. In addition, monocytes treated with nitrogen mustard

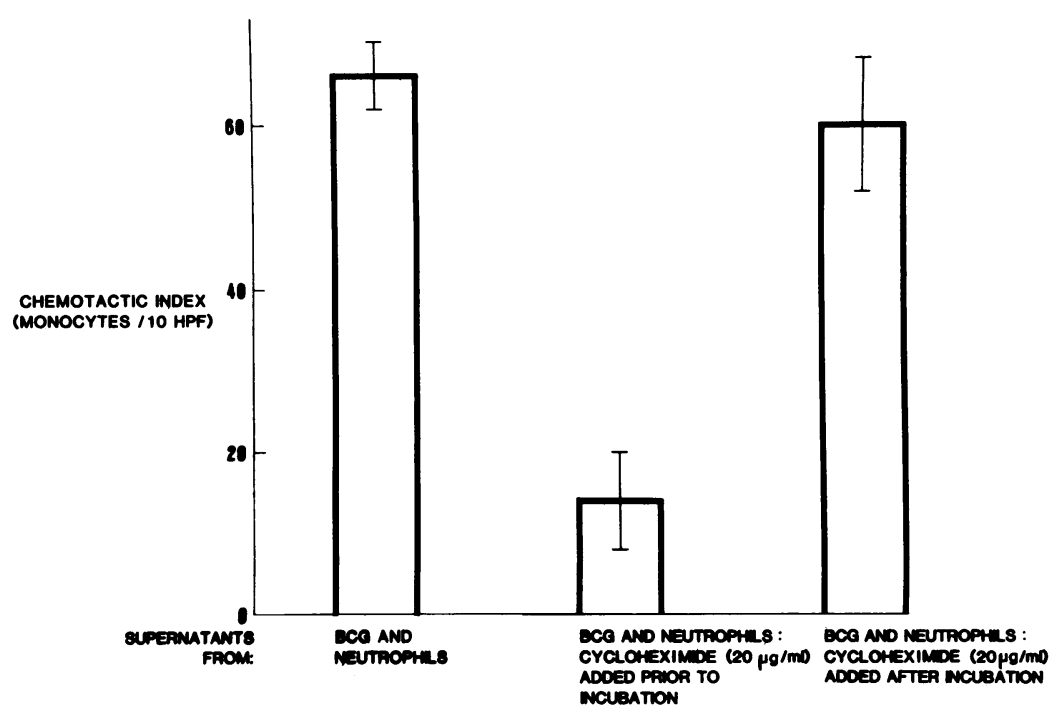

Figure 6. Effect of cycloheximide on monocyte chemotactic activity in supernatants from mixtures of neutrophils and BCG in vitro. Less $(P<0.05)$ chemotactic activity for monocytes was detected in supernatants from in vitro mixtures of BCG and neutrophils that were incubated in the presence of nontoxic concentrations of $20 \mu \mathrm{g} / \mathrm{ml}$ of cycloheximide. However, addition of cycloheximide $(20 \mu \mathrm{g} / \mathrm{ml})$ at the end of a 4-h incubation of BCG and neutrophils did not alter the chemotactic activity present in supernatants from mixtures of BCG and neutrophils in vitro. Each point is the mean $\pm 1 \mathrm{SE}$ of eight or more individual determinations. HPF, high power field. 


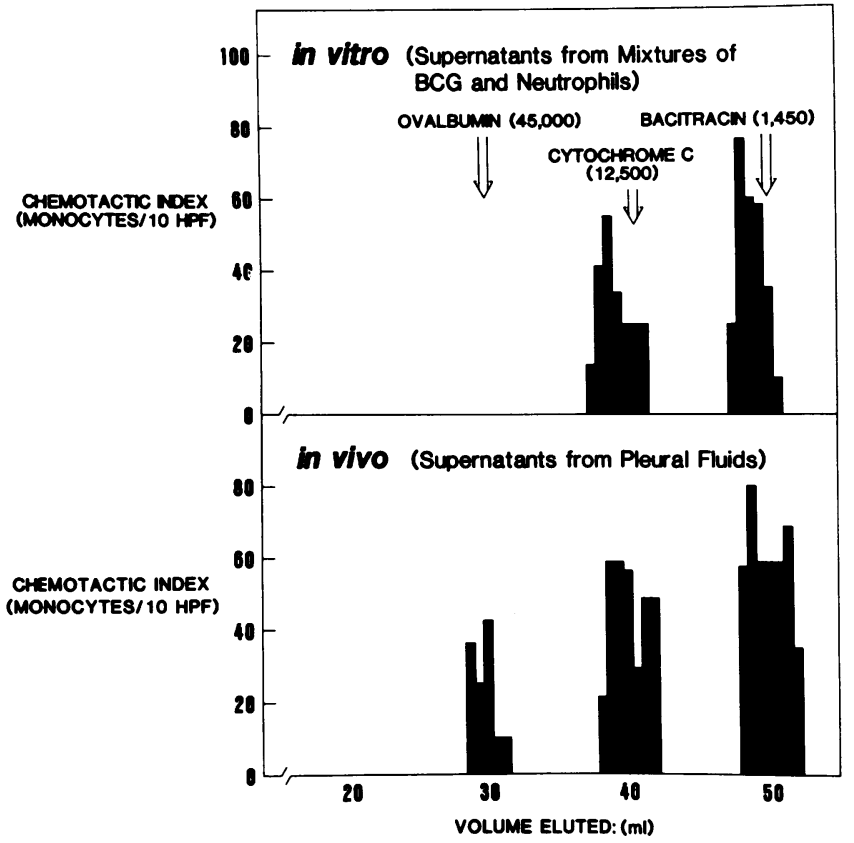

Figure 7. Molecular weights of monocyte chemotaxins in mixtures of neutrophils and BCG in vitro. Chemotaxins for monocytes from mixtures of BCG and neutrophils in vitro had molecular weights that were similar to chemotaxins for monocytes eluted from supernatants from pleural fluids of BCG-sensitized rabbits given intrapleural BCG. HPF, high power field.

in vitro responded normally to chemotactic stimuli in vitro. Therefore, because only neutrophils were injected into pleural spaces of neutropenic rabbits and because injection of neutrophils was followed by the appearance of monocytes, it appeared that neutrophils were the key component involved in recruitment of monocytes. This impression was substantiated by the following observations. First, increased chemotactic activity for monocytes was found in pleural fluids from normal and neutrophil-recon-

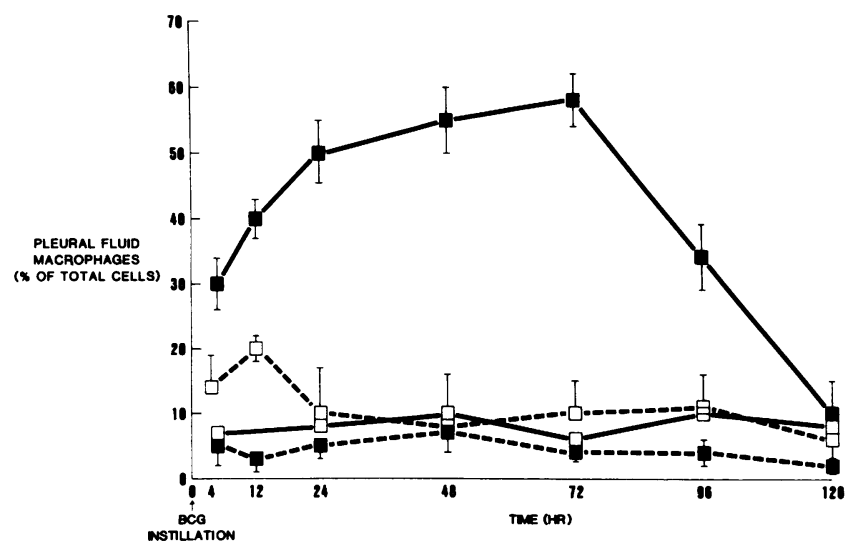

Figure 8. Accumulation of macrophages in pleural spaces of neutropenic BCG-sensitized rabbits injected intrapleurally with supernatants from mixtures of neutrophils and BCG in vitro. More $(P<0.05)$ macrophages were recovered from pleural fluids from neutropenic rabbits injected intrapleurally with supernatants from mixtures of BCG and neutrophils in vitro (--) than from rabbits injected with supernatants from mixtures of heat-killed neutrophils and BCG ( --- $)$, neutrophils $(\square--\square)$, or BCG ( $\square-\square)$ alone in vitro. Each point is the mean $\pm 1 \mathrm{SE}$ of eight or more individual determinations.

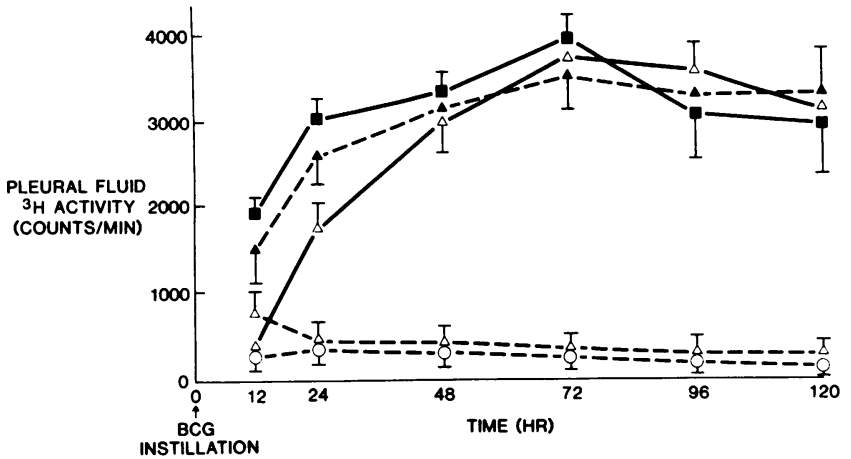

Figure 9. Pleural fluid cell pellet ${ }^{3} \mathrm{H}$ activity in BCG-sensitized rabbits previously injected intravenously with ${ }^{3} \mathrm{H}$-labeled monocytes $\left(2 \times 10^{7}\right.$ rabbit monocytes with 30,000 counts). ${ }^{3} \mathrm{H}$ counts were greater $(P<0.05)$ in cell pellets from pleural fluids from rabbits given intrapleural BCG $(\Delta-\Delta)$, neutropenic rabbits given intrapleural neutrophils, and BCG $(\Delta-\ldots-\Delta)$ or neutropenic rabbits given supernatants from mixtures of neutrophils and BCG in vitro (- - - ) than from neutropenic rabbits given BCG $\left(0--_{-}\right)$) or rabbits given saline $(\Delta--\Delta)$. Each point is the mean \pm 1 SE of six to eight individual determinations.

stituted neutropenic but not neutropenic rabbits given intrapleural BCG. Second, supernatants from mixtures of neutrophils and BCG in vitro contained chemotaxins for monocytes whose molecular weight resembled chemotaxins found in pleural fluids. Third, accumulation of monocytes occurred in pleural spaces of neutropenic rabbits injected with supernatants from in vitro mixtures of BCG and neutrophils but neither alone. Fourth, in experiments involving intravenous injection of tritiated thymidine-labeled monocytes, intrapleural injection of neutrophils and BCG or supernatants from mixtures of neutrophils and BCG was associated with an increase in pleural fluid cell pellet ${ }^{3} \mathrm{H}$ activity. These latter findings suggest that monocytes are being recruited from the peripheral blood and are consistent with other investigations using radiolabeling techniques which have shown that the pulmonary macrophage is recruited from the circulating pool of peripheral blood monocytes $(21,22)$. In aggregate, these investigations indicate that neutrophil-derived chemotaxins contribute to the recruitment of peripheral blood monocytes to pleural spaces of rabbits given BCG intrapleurally.

Previous studies have suggested that neutrophils might participate in recruitment of monocytes and the development of granuloma (23). For example, in sensitized hosts, influxes of neutrophils usually precede influxes of monocytes in tuberculous infection (24) and are often associated with a rapid resolution of infection (25). In contrast, in unsensitized hosts, decreased neutrophil responses are seen, granuloma formation is delayed and dissemination of infection is greater (26). Monocyte infiltration also occurs in control but not neutropenic rabbits given immune complexes (27). In addition, intratracheal injection of neutrophils causes accumulation of monocytes in the lung and immune complex-stimulated neutrophils generate chemotaxins for monocytes in vitro (28). Furthermore, decreased migration of mononuclear cells into Rebuck skin windows occurs when circulating neutrophils are decreased (29-31).

Neutrophils can produce factors in vitro that are selectively chemotactic for monocytes (32-36). One of these may be a low molecular weight factor present in neutrophil granules. In addition, in patients with specific granule deficiency, there appears 
to be an absence of neutrophil granule constituents that produce chemotactically active fragments of C5 (C5a and C5a des arg). It is presumed that this abnormality is responsible for the defective monocyte accumulation seen in these patients (37). Others have shown that a monocyte chemokinetic factor may be packaged in neutrophil-specific granules (38). In addition, it appears that active synthetic processes may also be involved in that inhibition of protein synthesis with cycloheximide reduced neutrophil production of chemotactic activity for monocytes. The latter is consistent with prior observations that neutrophils can synthesize proteins which are chemotactic for neutrophils (39). The 12,000-15,000-mol wt portion of the neutrophil-derived chemotaxin for monocytes appears different from C5a. First, cobra venom factor-treated, BCG-sensitized rabbits that subsequently received intrapleural BCG showed no decreases in pleural fluid volumes, cell counts, or chemotactic activities for monocytes in vitro. Second, addition of monospecific rabbit antiC5a serum did not inhibit monocyte chemotactic activities of supernatants from in vitro mixtures of neutrophils and BCG whereas addition of the same amounts of anti-C5a-treated serum inhibited monocyte (or neutrophil) chemotaxis toward human C5a or zymosan-activated serum in vitro. Third, the neutrophilderived monocyte chemotactic factor activity is also abolished by heating at $56^{\circ} \mathrm{C}$ for $30 \mathrm{~min}$ while $\mathrm{C} 5$ a activity is stable. Fourth, the neutrophil-derived monocyte chemotactic factor activity for neutrophils but not monocytes is decreased by serial dilution whereas serial dilutions of C5a maintain their chemotactic activity for both neutrophils and monocytes. Finally, the neutrophil-derived monocyte chemotactic factor elutes at $\sim 12,000$ $13,000 \mathrm{~mol} w t$ whereas while rabbit C5a has a mol wt of $\sim 15,000$.

The 12,000-15,000 mol wt neutrophil-derived monocyte chemotactic factor also does not appear to be crystal-induced chemotactic factor (39). First, the neutrophil-derived monocyte chemotactic factor is inactivated by heating at $56^{\circ} \mathrm{C}$ for $30 \mathrm{~min}$ whereas while crystal-induced chemotactic factor is stable when heated at $56^{\circ} \mathrm{C}$ for $30 \mathrm{~min}$. Second, the neutrophil-derived monocyte chemotactic factor is more active, even when diluted, for monocytes than neutrophils, whereas diluted crystal-induced chemotactic factor shows a greater degree of chemotactic activity for neutrophils than for monocytes. Finally, the larger portion of the neutrophil-derived monocyte chemotactic factor has a mol wt of 12,000-15,000 ( 50\% activity) and 1,000 (50\% activity) whereas crystal-induced chemotactic factor has a mol weight of 8,400 .

Our findings suggest a role for neutrophils in the recruitment of monocytes and the development of tuberculous granuloma. The mechanisms responsible for the initial recruitment of neutrophils were not investigated but may involve chemotaxins produced by interactions of BCG with serum or cells in pleural spaces. The final step in this cellular cascade may be engulfment and degradation of neutrophils by macrophages in that neutrophils containing BCG were seen within macrophages recovered from pleural spaces of BCG-sensitized rabbits given BCG intrapleurally (40).

\section{Acknowledgments}

The authors acknowledge the help of James T. Good, Jr., M.D., Ruth N. Harada, M.D., and Anna Tsang.

This work was supported in part by grants from the National Institutes of Health (HL24248, HL28182 and CA36752). The Colorado and
American Heart Associations, Colorado and American Lung Associations, the Council for Tobacco Research, USA Inc., and the Kroc, Swan, Hill, Kleberg, and R. J. Reynolds Foundations. Dr. V. B. Antony is the recipient of a New Investigator Award (A119384) from the National Institutes of Health; Dr. V. B. Antony and this work were recipients of the Cecil Lehman Meyer Research Award of the American College of Chest Physicians.

\section{References}

1. Adams, D. O. 1976. The granulomatous inflammatory response. Am. J. Pathol. 84:164-191.

2. Ebert, R. H., and H. W. Florey. 1939. The extravascular development of the monocyte observed in vivo. Br. J. Exp. Pathol. 20:342356.

3. Spector, W. G. 1969. The granulomatous inflammatory exudate. Int. Rev. Exp. Pathol. 8:1-55.

4. Altman, L. C. 1978. Chemotactic Lymphokines in Leukocyte Chemotaxis. J. I. Gallin and P. G. Quie, editors. Raven Press, New York. 267-287.

5. Jungi, T. W., and D. D. McGregor. 1979. Dissociation of macrophage accumulation and local chemotactic activity in delayed inflammation sites. Cell Immunol. 47:274-284.

6. Montgomery, L. G., and W. S. Lemon. 1933. The cellular reaction of the pleura to infection with mycobacteria tuberculosis. $J$. Thoracic Surg. 2:429-439.

7. Repine, J. E., and C. C. Clawson. 1978. Influence of surface proteins and separation techniques on neutrophil unstimulated or stimulated locomotion in vitro. J. Reticuloendothel. Soc. 24:217-226.

8. Repine, J. E., J. G. White, C. C. Clawson, and B. M. Holmes. 1974. Effects of phorbol myristate acetate on the metabolism and ultrastructure of neutrophils in chronic granulomatous disease. J. Clin. Invest. 54:83-89.

9. Boyum, A. 1968. Isolation of mononuclear cells and granulocytes from human blood. Scand. J. Clin. Invest. 21(Suppl. 97):77-89.

10. Repine, J. E., C. C. Clawson, and P. S. Friend. 1977. Influence of a deficiency of the second component of complement on the bactericidal activity of neutrophils in vitro. J. Clin. Invest. 59:802-809.

11. Lurie, M. B. 1928. The fate of human and bovine tubercle bacilli in various organs of the rabbit. J. Exp. Med. 48:155-182.

12. Lurie, M. B. 1929. The fate of tubercle bacilli on the organs of reinfected rabbits. J. Exp. Med. 50:747-765.

13. Good, J. T., Jr., D. A. Taryle, T. M. Hyers, and S. A. Sahn. 1978. Clotting and fibrinolytic activity of pleural fluid in a model of pleural adhesions. Am. Rev. Respir. Dis. 118:903-908.

14. Sahn, S. A., and D. E. Potts. 1978. Turpentine pleurisy in rabbits: A model of pleural fluid acidosis and low pleural fluid glucose. Am. Rev. Respir. Dis. 118:893-901.

15. Sahn, S. A., D. A. Taryle, and J. T. Good, Jr. 1979. Experimental empyema: Time course and pathogenesis of pleural fluid acidosis and low pleural fluid glucose. Am. Rev. Respir. Dis. 120:355-361.

16. Stauffer, J. E., D. E. Potts, and S. A. Sahn. 1978. Cellular content of the normal rabbit pleural space. Acta Cytol. 22:570-574.

17. Shasby, D. M., R. B. Fox, R. N. Harada, and J. E. Repine. 1982. Reduction of the edema of acute hyperoxic lung injury by granulocyte depletion. J. Appl. Physiol. 52:1237-1244.

18. Clawson, C. C., and J. E. Repine. 1976. Quantitation of maximal bactericidal capability in human neutrophils. J. Lab. Clin. Med. 88:316327.

19. Hunninghake, G. W., J. I. Gallin, and A. S. Fauci. 1978. Immunologic reactivity of the lung. The in vivo and in vitro generation of a neutrophil chemotactic factor by alveolar macrophages. Am. Rev. Respir. Dis. 117:15-23.

20. Zigmond, S. H., and J. G. Hirsch. 1971. Leukocyte locomotion and chemotaxis: new method for evaluation and demonstration of a cell derived chemotactic factor. J. Exp. Med. 136:2645-2654.

21. Blusse' Van Ovd Alblas, A., B. Van Der Linden Schrever, and R. Van Furth. 1981. Origin and kinetics of pulmonary macrophages 
during an inflammatory reaction induced by intravenous administration of heat killed bacillus Calmette Guérin. J. Exp. Med. 154:235-252.

22. Blusse' Van Ovd Alblas, A., B. Van Der Linden Schrever, and R. Van Furth. 1983. Origin and kinetics of pulmonary macrophages during an inflammatory reaction induced by intraalveolar administration of aerolyzed heat killed BCG. Am. Rev. Respir. Dis. 276-281.

23. Paterson, R. C. 1917. The pleural reaction to innoculation with tubercle bacilli in vaccinated and normal guinea pigs. Am. Rev. Tuberc. 1:353-371.

24. Pinner, M. 1928. A note on exudative and productive processes in pleural tuberculous infection. Am. Rev. Tuberc. 17:637-733.

25. Lemon, W. S., and W. H. Feldman. 1943. Experimental tuberculous pleural effusion. Am. Rev. Tuberc. 48:177-183.

26. Rich, A. R. 1951. The Pathogenesis of Tuberculosis. Charles C Thomas, Springfield, Illinois.

27. Ordal, J. C., and R. B. Dreisen. 1980. The effect of neutrophils on the accumulation of mononuclear phagocytes in immune complexinduced alveolitis. Chest. 78:534 (abstract).

28. Henson, P. M. 1971. Interaction of cells with immune complexes: adherence, release of constituents and tissue injury. J. Exp. Med. 134(Suppl.):114-135.

29. Rebuck, J. W. Cytology of acute inflammation in man as demonstrated by two original technical procedures with particular reference to the role of lymphocytes. Ph.D. thesis. University of Minnesota, Minneapolis.

30. Page, A. R., and R. A. Good. 1957. Studies on cyclic neutropenia Am. J. Dis. Child. 94:623-661.

31. Dale, D. C., and J. M. Wolff. 1971. Skin window studies of the acute inflammatory responses of neutropenic patients. Blood. 38:138142.
32. Ward, P. A. 1968. Chemotaxis of mononuclear cells. J. Exp. Med. 128:1201-1221.

33. Wintroub, B. U., L. B. Klickstien, C. E. Kaempfer, and K. F. Austen. 1981. A human neutrophil-dependent pathway for generation of angiotensin II: purification and physicochemical characterization of the plasma protein substrate. Proc. Natl. Acad. Sci. USA. 78:1203-1208.

34. Ward, P. A., and J. H. Hill. 1970. C5 chemotactic fragments produced by an enzyme in lysosomal granules of neutrophils. J. Immunol. 104:535-543.

35. Spilberg, I., and J. Mehta. 1979. Demonstration of a specific neutrophil receptor for a cell-derived chemotactic factor. J. Clin. Invest. 63:85-88.

36. Goetzl, E. J., L. B. Klickstein, K. W. K. Watt, and B. U. Wintroub. 1980. The preferential human mononuclear leukocyte chemotactic activity of the substituent tetrapeptides of angiotensin II. Biochem. Biophys. Res. Commun. 97:1097-1102.

37. Gallin, J. I., M. P. Fletcher, B. E. Seligmann, S. Hoffstein, K. Lehrs, and N. Mounessa. 1982. Human neutrophil specific granule deficiency: a model to assess the role of neutrophil specific granules in the evolution of the inflammatory response. Blood. 59:1317-1329.

38. Wright, D. G., and D. Greenwald. 1979. Increased motility and maturation of human blood monocytes stimulated by products released from neutrophil secondary granules. Blood. 54:95 (abstract).

39. Spilberg, I., B. Mandell, and R. D. Wochner. 1974. Studies on crystal induced chemotactic factor. I. Requirement for protein synthesis and neutral protease activity. J. Lab. Clin. Med. 83:56-63.

40. Antony, V. B., J. E. Repine, R. N. Harada, J. T. Good, and S. A. Sahn. 1983. Inflammatory responses in experimental tuberculous pleurisy. Acta Cytol. 27:355-361. 